\title{
Workplace Bullying Among Pediatric Nurses: Its Relationship to Children Exposure to Some Adverse Events and Parents Satisfaction
}

\author{
Dr. Amal Abderazik Fathalla ${ }^{1} \quad$ Dr. Rehab Abd Allah Nassar ${ }^{2}$ \\ 1.Lecturer of Pediatric Nursing Department, Faculty of Nursing, Menofia University. Egypt \\ 2.Lecturer of Nursing Administration, Faculty of Nursing, Menofia University. Egypt
}

\begin{abstract}
:
Background: Workplace bullying especially in healthcare setting and among nurses is a widespread and serious problem. A number of countries including Egypt have increasingly been focusing on exploring this problem in order to prevent its negative implications on the victim's health and work performance. The purpose of this study was to determine relationship between workplace bullying among pediatric nurses and children exposure to some adverse events and parents satisfaction. A correlational research design was used. The study was conducted at hemodialysis unit, Pediatric Intensive Care Unit (PICU), Neonatal Intensive Care Unit (NICU) and Pediatrics Department. A convenience sample of 118 staff nurses and random sample of 100 children parents were assigned from the previously mentioned settings. Three tools were used: Workplace Bullying Behaviors Scale, Parent's Satisfaction Questionnaire and Children Adverse Events Scale. The results of this study showed that, the highest percentage of the studied nurses in different studied areas had moderate level of workplace bullying behavior's exposure (61.0\%), children had moderately level of exposure to some adverse events $(61.9 \%)$ as reported by the studied staff nurses' and the highest percentage of the studied parents have moderately level of satisfaction of nursing care that was provided to their children (45\%). The study concluded that, there was a positive relationship between the exposure of the nurses to the behaviors of bullying and the exposure of children to the adverse events. Also, there was a negative correlation between the exposure of nurses to bullying behavior and the level of parents 'satisfaction with the care given to their children. So, it was recommended that identifying behaviors of bullying and raising awareness of bullying behavior in society should be the first step in the solution. Subsequently, in-service training programs on bullying behaviors in all pediatric units should be designed and implemented to improve the knowledge and practices of nurses to control and prevent bullying in the workplace.
\end{abstract}

Keywords: Workplace Bullying, children adverse events, and Parents Satisfaction

DOI: $10.7176 / \mathrm{JHMN} / 58-07$

\section{Introduction}

Workplace bullying in the healthcare profession has been documented as a problem for healthcare institutions since the $1980^{[1]}$. Workplace bullying is a major dilemma for nurses in the hospital. In a recent sentinel events alert, the seriousness of workplace bullying publicizing numerous negative penalties that can prevented when bullying behaviors were controlled ${ }^{[2]}$. The concept of being bullied is subjective in nature and is derived from how one perceives the situation. There have been some Contradictions around the definition of workplace bullying. In many studies, the term workplace bullying has been used synonymously with the term horizontal hostility. Horizontal hostility is defined as bullying behavior among peers or co-workers on the same status within an organization ${ }^{[3]}$.

Bullying can be defined as the continuous and normally intentional misuse of power or position to intimidate, humiliate or undermine. In general, bullying entails the behavior targeted at a person to humiliate and stigmatize socially. It also aims at sabotaging the victim's reputation by attacking the victim's character and professional aptitude. A person can experience bullying at work from managers, supervisors, co-workers or subordinates ${ }^{[4]}$. Furthermore, it includes accusations of incompetence despite a history of excellence in the area of practice, gossiping about co-workers, concealment of information which is important to patient care, continuous feelings of stress and fear of additional bullying events, being told by a supervisor to "get tougher skin" or "work out your differences" when approached about bullying, and screaming or yelling at a fellow worker in front of others to make him/her look bad ${ }^{[5]}$.

There are many factors that enhance the risk for bullying behavior which include the following: Significant organizational change (e.g., major internal restructuring, technological change), Worker characteristics (e.g., age, gender, parental status, apprentice or trainee), Workplace relationships (e.g., scarce information flow between organizational levels, lack of employee involvement in decisions and work systems (e.g., lack of policies about behavior, high rate and intensity of work, staff shortages, interpersonal conflict, organizational constraints, role ambiguity, and role conflict ${ }^{[6]}$.

Nurses in pediatric departments and units are the most common nurses that can exposure for workplace 
bullying. This is due to, these nurses endure hard work that requires them to provide full nursing care, ensure the protection, safety and prevent medical errors that may occur within the unit and also provide assistance to parents. And bullying here in such units and among these nurses may lead to a negative consequences on the quality of nursing services and children safety. Apart from its high prevalence rate worldwide, workplace bullying has highly impact on the nurses physical and emotional health, which could in turn affect a hospitals function and, ultimately the quality of care provided to its patients ${ }^{[7]}$.

Bullying among pediatric nurses is often paired with poor management styles or lack of presence of pediatric management manger in a pediatric department or unit. Pediatric nursing managers need to be aware of the impact that bullying has on their staff nurses. It is important to be aware of potential signs and symptoms accompanied with bullying in a workplace which include: Grievances by nurses against their manager, declining work performance of dedicated and hard-working nurses, increased stress and tensions between staff in a unit, poor morale, reported fear of a co-worker by other workers, individual symptoms of depression and increased absenteeism in a department/unit ${ }^{[8,9]}$. in addition, , The hostility that stems from workplace bullying among pediatrics nurses can corresponding to medical errors, patient dissatisfaction, and increased healthcare costs in their units. Studies have shown that there is also a correlation between nursing retention rates, negative outcomes for nurses on a personal level, and impact negatively on patients and the healthcare system and workplace bullying ${ }^{[2][10]}$.

Indeed, research has related workplace bullying to some adverse events (care giving errors, unsafe medication practices in nursing settings, nosocomial infection and fall with injuries and poor of parent satisfaction. Medical errors referred as incidents, accidents and adverse events (AEs), can cause rigorous harm even leading to death ${ }^{[11]}$. Adverse events can be defined as instances which indicate that a patient has received poor quality care. Adverse events that result due to workplace bullying appear to be ever present and inevitable in highly complex and technological driven environments as Neonatal Intensive Care Units (NICUs), Pediatric Intensive Care Units (PICUs) and Hemodialysis units. Although, these units as a highly complex, stressful, technology driven environment, it provide multidisciplinary care in critically ill children and newborn and pediatric nurses should serve the goal of longer survival and satisfactory quality of life ${ }^{[12][13]}$. In these units compared to adults units in a hospital, errors occur 8 times more than often, raising the chance to cause rigorous harm. Specifically, medication errors lead to harm at a range of $4-27 \%{ }^{[14,15]}$.

For parents, the children hospitalization experience is an unexpected journey filled with stress, emotional turmoil, strains on relationships and sometimes depression ${ }^{[16]}$. They hardly ever feel safe from the fear and doubt of the problems that can occur while the child is in care . In pediatrics units' journey has many unpredicted ups, downs, and turns of event ${ }^{[16,17]}$. Hence, usually pediatric nurses provides transparent communication, nonverbal communication and continuity of care are among the concepts of family centered care and palliative care $[18,19]$. So, workplace bullying among these nurses became more greatly pressured, depressed and stressed. This leads to direct negative impact on the relationship of these nurses with parents. With these pressures, these nurses become less flexible in dealing with parents and do not give them enough chance to ask about their children ${ }^{[20,21]}$.

It is logical to expect that work environments in which bullying are widespread are not conducive to a positive patient safety climate and may therefore be associated with higher patient safety risk. Indeed, many researches have related workplace bullying to caregiving errors and unsafe medication practices in nursing settings. However, we could find no studies linking between workplace bullying to exposure some adverse events that effect on children safety and parents satisfaction of care provided for their children ${ }^{[22,23,24]}$. So, the purpose of this study was to determine relationship between workplace bullying among pediatric nurses and exposure of children to some adverse events and parents satisfaction.

\section{Significant of study:}

Bullying not only demeans and humiliates a colleague; it also negatively affects the patient outcomes. It increases occupational stress and increase staff turnover. On other hand, increase workplace bullying between nurses can increase in patient safety risks and medication errors. In a study by Thomas \& Burk (2009), many nursing staff reported feeling unwanted, ignored, mistrusted, disbelieved, unfairly blamed and publicly humiliated during their clinical rotations. It also, effect on their performances and their patient and caregivers relationship ${ }^{[25]}$. In addition, $34 \%$ of nurses who are bullied considered leaving the profession altogether and $36 \%$ of calls to the Workplace Bullying Institute are from nurses, more than any other profession. Also, $48 \%$ of graduate nurses are scared of experiencing workplace bullying, $60 \%$ of new nurses leave their first job within six months because of some form of verbal abuse or treatment from a colleague and $85 \%$ of nurses have been verbally abused by another nurse. As known, nursing is a tough profession, and it becomes worse by workplace bullying behaviors that nurses expose to ${ }^{[26]}$. So, it is the time to assess, monitor workplace bullying behaviors and its relationship to children exposure to some adverse events and parents satisfaction. 


\subsection{Purpose of the study:}

The purpose of this study was to determine relationship between workplace bullying among pediatric nurses and children exposure to some adverse events and parents satisfaction.

\subsection{Research questions:}

1. What is the level of workplace bullying among pediatric staff nurses at selected setting?

2. What is nurses' agreement regarding children exposure to some of adverse events at selected setting?

3. What is the level of parent's satisfaction of the nursing care provided to their children at selected setting?

4. Is there a relation among workplace bullying, children exposure to some of adverse events and parent satisfaction at selected setting?

\section{Subjects and Methods}

\subsection{Research design:}

A correlational research design was used in conducting the present study.

\subsection{Setting:}

The study was conducted at pediatric units in Menoufia University Hospital at Shebin El Kom city, Menoufia Governorate at Pediatric Intensive Care Unit (PICUs), Neonate Intensive Care Unit (NICU), Pediatric Hemodialysis unit and Pediatrics Department.

\subsection{Sample:}

The subjects of the present study were classified into two groups:

- The first group consisted of a convenience sample of (118) pediatric nurses all available in the previously mentioned areas ( 30 in hemodialysis unit, 30 in PICU, 37 in NICU and 18 pediatrics department) who agree in participating in the study and have at least one year experience in their current department.

- The second group consisted of 100 children parents were randomly selected from the previously mentioned areas who accept to participate in this study and can read and write.

\subsection{Tools of data collection:}

Three tools were utilized in the current study to collect the data:

2.4.1: Tool one: Workplace Bullying Behaviors Scale.

This tool was developed by Samuels, (2015) to determine work place bullying characteristics ${ }^{[27]}$. It was divided into two parts:

Part one: Socio- demographic Characteristics of Nurses. It included the following items: age; unit; years of experience; marital status and educational qualifications.

Part two: Workplace Bullying Behaviors Scale.

It included of 22 items aimed to determine nurse's exposure to workplace bullying behaviors. (e.g. Someone withholding information which affects your performance, Being humiliated or ridiculed in connection with your work,........). Each item is rated from 1 to 5 in which $\mathbf{1}$ indicating strongly disagrees; 2 indicating disagree; 3 indicating Neutral; 4 indicating Agree and 5 indicating strongly agree. Scoring system: High score should correspond to high level of nurse's exposure to workplace bullying behaviors. Total score of the scale was 110 . If the nurses had score less than (66) this indicated that their exposure to low level of workplace bullying behaviors, if the nurses had score (more than 66 and less than 82) this indicate that the nurses had moderately exposed to workplace bullying, while, if the nurses score was (more 82) this indicate that the nurses had highly exposed to workplace bullying.

\subsection{2: Tool two: Parent's satisfaction questionnaire}

It was developed by Koontz, (2003) and modified by the researcher to measure the parents satisfaction about nursing care provided to their children in different pediatric units ${ }^{[28]}$. It included of 14 items. Total score of the scale was (28). If the score of the participant less than (17) this indicated low level of parents satisfaction, if the score of the parents score is (more than 18 and less than 21) this indicate that the parents were moderately satisfied while, if the participants' score is (more 21) this indicate that the parents were highly satisfied.

\subsection{3: Tool three: Children Adverse Events Scale.}

It was developed by Sochalski, (2001) derived from the American Nurses Association Nursing Quality Indicators, (2000). It involved of 5 items that assess agreement of nurses' regarding some children exposure to some adverse events (children received wrong medication or dose, nosocomial infections, falls, fall with injury, and complaints from parents and family ${ }^{[29,30]}$. Total score of the scale was (25). If the score less than (15) this indicated that children exposure to lowest level of adverse events, if children had score (more than 15 and less than 18) this indicate that the children had moderately exposed to adverse events, while, if the children score was 
(more 18) this indicate that the children had highly adverse events.

\subsection{Content validity and reliability:}

2.5.1. Validity Bilingual group of five experts was selected to test the content and face validity of the tools. Necessary modifications and deleting of some questions were done to reach the final valid version of the tools. The tools were considered valid from the experts' perspective. 2.5.2. Reliability of the tools was tested to reliability by measuring their internal consistency using Cronbach's alpha coefficient method. This turned to be Workplace Bullying Behaviors Scale $(\mathrm{a}=.420)$; parents satisfaction scale $(\mathrm{a}=.616)$ and Children Adverse Events Scale $(\alpha=.568)$. Thus, this indicates a high degree of reliability for the study tools.

\subsection{Administrative approval:}

An official permission was obtained from the Dean of Faculty of Nursing, Menofia University to conduct the study. The letter contained the title, aim of the study and method of data collection. The actual data collection took 3 months beginning from the first of June until the end of August 2018.

2.7. Pilot study: Before collecting the data, the research questionnaires administered to $10 \%$ of the study sample (12 pediatric nurses and 10 children parents) to test clarity of the tools. Many changes were done on many statements based on the results of the pilot study. Nurses and children parents who included in the pilot study excluded from data analysis. Additionally, pilot study done to estimate time needed for each nurse and each parent to complete the tools. The first one required 10-15 minutes, while, second and third tools required 10 minutes to be completed.

\subsection{Data collection procedure:}

The data were collected in the morning and afternoon shift for a period of 3 months from staff nurses and children parents through using the previously mentioned tools. After data were collected then statistical analyses by expertise.

\subsection{Statistical Analysis:}

Data was coded and transformed into specially designed form to be suitable for computer entry process. Data was entered and analyzed by using SPSS (Statistical Package for Social Science) statistical package version 16. Graphics were done using Excel program. Quantitative data were expressed as mean \& standard deviation $(\overline{\mathbf{x}} \pm$ SD) and analyzed by applying t-test for comparison of two groups of normally distributed variables. Qualitative data were expressed as number and percentage (No \& \%).It was and analyzed by using chi-square test $\left(\mathrm{X}^{2}\right)$ for $2 \mathrm{X} 2$ table, Pearson correlation was used for explaining relationship between normally distributed quantitative variable.

\section{Result:}

Table (1): Distribution of the studied nurses according to their demographic characteristics $(\mathrm{n}=118)$

\begin{tabular}{|c|c|c|c|c|c|c|c|c|c|c|}
\hline \multirow{3}{*}{$\begin{array}{l}\text { Demographic } \\
\text { Characteristics }\end{array}$} & \multicolumn{8}{|c|}{ Unit } & & \\
\hline & \multicolumn{2}{|c|}{$\begin{array}{l}\text { Hemodialysis } \\
(\mathrm{n}=33)\end{array}$} & \multicolumn{2}{|c|}{$\begin{array}{l}\text { Premature } \\
(\mathrm{n}=37)\end{array}$} & \multicolumn{2}{|c|}{$\begin{array}{l}\text { PICU } \\
(n=30)\end{array}$} & \multicolumn{2}{|c|}{$\begin{array}{l}\text { Pediatric department } \\
(\mathrm{n}=18)\end{array}$} & \multicolumn{2}{|c|}{$\begin{array}{l}\text { Total } \\
(n=118)\end{array}$} \\
\hline & No & $\%$ & No & $\%$ & No & $\%$ & No & $\%$ & No & $\%$ \\
\hline Age/ years & & & & & & & & & & \\
\hline $20-<30$ & 14 & 42.4 & 8 & 21.6 & 5 & 16.7 & 3 & 16.7 & 30 & 25.4 \\
\hline $30-<40$ & 17 & 51.5 & 25 & 67.6 & 23 & 76.7 & 12 & 66.7 & 77 & 65.3 \\
\hline $40-<50$ & 2 & 6.1 & 4 & 10.8 & 2 & 6.7 & 3 & 16.7 & 11 & 9.3 \\
\hline Years of experience: & & & & & & & & & & \\
\hline $1-<5$ & 12 & 36.4 & 7 & 18.9 & 5 & 16.7 & 3 & 16.7 & 27 & 22.9 \\
\hline $5-<10$ & 18 & 54.5 & 22 & 59.5 & 17 & 56.7 & 11 & 61.1 & 68 & 57.6 \\
\hline $10-<15$ & 3 & 9.1 & 5 & 13.5 & 7 & 23.3 & 1 & 5.6 & 16 & 13.6 \\
\hline$>15-$ & 0 & 0.0 & 3 & 8.1 & 1 & 3.3 & 3 & 16.7 & 7 & 5.9 \\
\hline Marital status: & & & & & & & & & & \\
\hline Single & 11 & 33.3 & 6 & 16.2 & 3 & 10.0 & 0 & 0.0 & 20 & 16.9 \\
\hline Married & 22 & 66.7 & 31 & 83.8 & 27 & 90.0 & 18 & 100.0 & 98 & 83.1 \\
\hline
\end{tabular}


Table 1 shows distribution of the studied nurses according to their demographic characteristics. Approximately two thirds (65.3\%) of nurses were $30-<40$ years old. More than half of nurses $(57.6 \%)$ had 5$<10$ years of experience. The majority of them $(83.1 \%)$ were married.

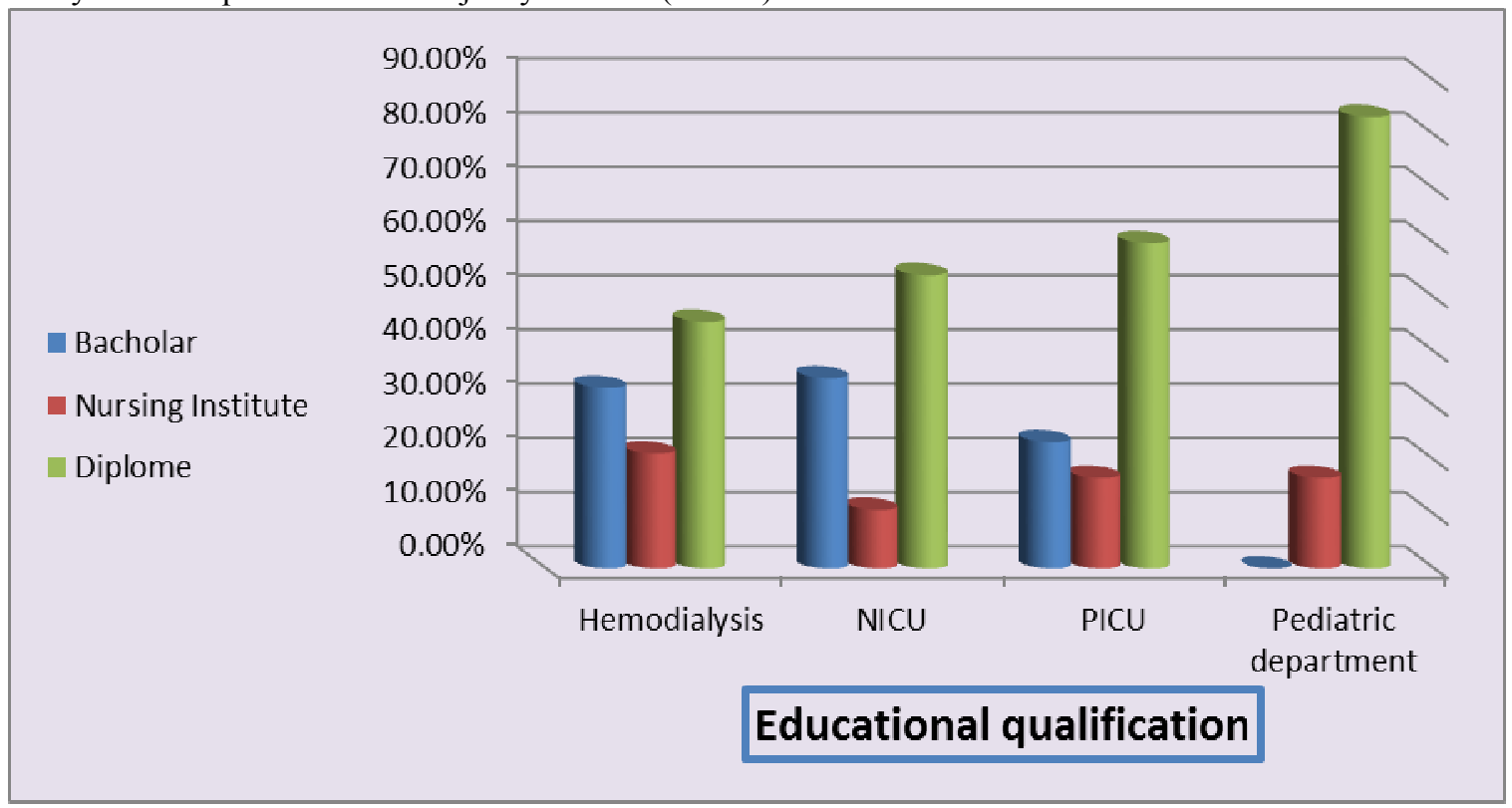

Figure (1): Distribution of the studied staff nurses according to their educational level (n=118)

Figure 1 clarifies the distribution of the studded staff nurses according to their educational level. It was clear that the highest percentage of the studied staff nurses in different studied areas (Hemodialysis, NIC, PICU, and Pediatric department) had nursing diplome (45.5\%, 54.1\%, 60.0\%, and 83.3\% respectively).

Table (2): Distribution of nurses according to their exposure to workplace bullying behaviors in different studied areas departments- unites $(n=1)$

\begin{tabular}{|c|c|c|c|c|c|c|c|c|c|c|c|c|}
\hline \multirow[t]{3}{*}{ Items } & & \multicolumn{8}{|c|}{ units } & \multirow{2}{*}{\multicolumn{2}{|c|}{$\begin{array}{r}\text { Total } \\
(\mathrm{n}=118)\end{array}$}} & \multirow[b]{3}{*}{$\mathbf{X}^{2}$} \\
\hline & & \multicolumn{2}{|c|}{$\begin{array}{l}\text { Hemodialysis } \\
\quad(n=33)\end{array}$} & \multicolumn{2}{|c|}{$\begin{array}{c}\text { Preterm } \\
(\mathrm{n}=37)\end{array}$} & \multicolumn{2}{|c|}{$\begin{array}{l}\text { PICU } \\
(n=30)\end{array}$} & \multicolumn{2}{|c|}{$\begin{array}{c}\text { Pediatric } \\
\text { department } \\
(\mathbf{n}=18)\end{array}$} & & & \\
\hline & & No & $\%$ & No & $\%$ & No & $\%$ & No & $\%$ & No & $\%$ & \\
\hline \multirow{2}{*}{$\begin{array}{l}\text { Someone withholding } \\
\text { information which affects } \\
\text { your performance }\end{array}$} & strongly disagree & 16 & 48.5 & 7 & 18.9 & 0 & 0.0 & 0 & 0.0 & 23 & 19.5 & \multirow[t]{2}{*}{$29.30^{* *}$} \\
\hline & disagree & 17 & 51.5 & 30 & 81.1 & 30 & 100.0 & 18 & 100.0 & 95 & 80.5 & \\
\hline \multirow{4}{*}{$\begin{array}{l}\text { Being humiliated or } \\
\text { ridiculed in connection } \\
\text { with your work }\end{array}$} & strongly disagree & 7 & 21.2 & 8 & 21.6 & 1 & 3.3 & 2 & 11.1 & 18 & 15.3 & \multirow{4}{*}{$13.76^{\mathrm{ns}}$} \\
\hline & disagree & 13 & 39.4 & 22 & 59.5 & 20 & 66.7 & 12 & 66.7 & 67 & 56.8 & \\
\hline & Neutral & 12 & 36.4 & 5 & 13.5 & 9 & 30.0 & 4 & 22.2 & 30 & 25.4 & \\
\hline & Agree & 1 & 3.0 & 2 & 5.4 & 0 & 0.0 & 0 & 0.0 & 3 & 2.5 & \\
\hline \multirow{5}{*}{$\begin{array}{l}\text { Being ordered to do } \\
\text { work below your level of } \\
\text { competence }\end{array}$} & strongly disagree & 1 & 3.0 & 3 & 8.1 & 1 & 3.3 & 0 & 0.0 & 5 & 4.2 & \multirow{5}{*}{$36.55^{* *}$} \\
\hline & disagree & 3 & 9.1 & 12 & 32.4 & 1 & 3.3 & 4 & 22.2 & 20 & 16.9 & \\
\hline & Neutral & 17 & 51.5 & 6 & 16.2 & 23 & 76.7 & 4 & 22.2 & 50 & 42.4 & \\
\hline & Agree & 7 & 21.2 & 12 & 32.4 & 5 & 16.7 & 7 & 38.9 & 31 & 26.3 & \\
\hline & strongly agree & 5 & 15.2 & 4 & 10.8 & 0 & 0.0 & 3 & 16.7 & 12 & 10.2 & \\
\hline \multirow{5}{*}{$\begin{array}{l}\text { Having key areas of } \\
\text { responsibility removed or } \\
\text { replace with more trivial } \\
\text { or unpleasant tasks. }\end{array}$} & strongly disagree & 1 & 3.0 & 6 & 16.2 & 0 & 0.0 & 0 & 0.0 & 7 & 5.9 & \multirow{5}{*}{$36.84^{* *}$} \\
\hline & disagree; & 4 & 12.1 & 3 & 8.1 & 5 & 16.7 & 1 & 5.6 & 13 & 11.0 & \\
\hline & Neutral & 22 & 66.7 & 5 & 13.5 & 9 & 30.0 & 7 & 38.9 & 43 & 36.4 & \\
\hline & Agree & 4 & 12.1 & 15 & 40.5 & 10 & 33.3 & 9 & 50.0 & 38 & 32.2 & \\
\hline & strongly agree & 2 & 6.1 & 8 & 21.6 & 6 & 20.0 & 1 & 5.6 & 17 & 14.4 & \\
\hline \multirow{5}{*}{$\begin{array}{l}\text { Spreading of gossip and } \\
\text { rumours about you. }\end{array}$} & strongly disagree & 5 & 15.2 & 13 & 35.1 & 5 & 16.7 & 9 & 50.0 & 32 & 27.1 & \multirow{5}{*}{$25.80^{* *}$} \\
\hline & disagree; & 16 & 48.5 & 16 & 43.2 & 17 & 56.7 & 6 & 33.3 & 55 & 46.6 & \\
\hline & Neutral & 12 & 36.4 & 3 & 8.1 & 8 & 26.7 & 2 & 11.1 & 25 & 21.2 & \\
\hline & Agree & 0 & 0.0 & 3 & 8.1 & 0 & 0.0 & 1 & 5.6 & 4 & 3.4 & \\
\hline & strongly agree & 0 & 0.0 & 2 & 5.4 & 0 & 0.0 & 0 & 0.0 & 2 & 1.7 & \\
\hline
\end{tabular}




\begin{tabular}{|c|c|c|c|c|c|c|c|c|c|c|c|c|}
\hline \multirow[t]{3}{*}{ Items } & & \multicolumn{8}{|c|}{ units } & \multirow{2}{*}{\multicolumn{2}{|c|}{$\begin{array}{r}\text { Total } \\
(n=118)\end{array}$}} & \multirow[b]{3}{*}{$\mathbf{X}^{2}$} \\
\hline & & \multicolumn{2}{|c|}{$\begin{array}{l}\text { Hemodialysis } \\
\qquad(\mathrm{n}=\mathbf{3 3})\end{array}$} & \multicolumn{2}{|c|}{$\begin{array}{l}\text { Preterm } \\
(\mathrm{n}=37)\end{array}$} & \multicolumn{2}{|c|}{$\begin{array}{l}\text { PICU } \\
(n=30)\end{array}$} & \multicolumn{2}{|c|}{$\begin{array}{c}\text { Pediatric } \\
\text { department } \\
(\mathrm{n}=18)\end{array}$} & & & \\
\hline & & No & $\%$ & No & $\%$ & No & $\%$ & No & $\%$ & No & $\%$ & \\
\hline \multirow{5}{*}{ 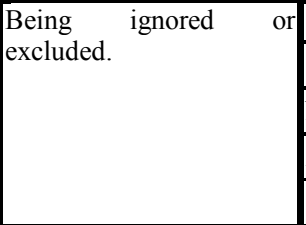 } & strongly disagree & 2 & 6.1 & 3 & 8.1 & 2 & 6.7 & 1 & 5.6 & 8 & 6.8 & \multirow{5}{*}{$23.36^{\mathrm{ns}}$} \\
\hline & disagree; & 8 & 24.2 & 8 & 21.6 & 1 & 3.3 & 3 & 16.7 & 20 & 16.9 & \\
\hline & Neutral & 23 & 69.7 & 15 & 40.5 & 19 & 63.3 & 14 & 77.8 & 71 & 60.2 & \\
\hline & Agree & 0 & 0.0 & 7 & 18.9 & 5 & 16.7 & 0 & 0.0 & 12 & 10.2 & \\
\hline & strongly agree & 0 & 0.0 & 4 & 10.8 & 3 & 10.0 & 0 & 0.0 & 7 & 5.9 & \\
\hline \multirow{2}{*}{$\begin{array}{l}\text { Having insulting or } \\
\text { offensive remarks made } \\
\text { about your person (i.e. } \\
\text { habits and background), } \\
\text { your attitudes or your } \\
\text { private life. }\end{array}$} & strongly disagree & \multirow[t]{2}{*}{12} & \multirow[t]{2}{*}{36.4} & \multirow[t]{2}{*}{14} & \multirow[t]{2}{*}{37.8} & \multirow[t]{2}{*}{15} & \multirow[t]{2}{*}{50.0} & \multirow[t]{2}{*}{7} & \multirow[t]{2}{*}{38.9} & \multirow[t]{2}{*}{48} & 40.7 & \\
\hline & disagree; & & & & & & & & & & 59.3 & $1.48^{\mathrm{ns}}$ \\
\hline Being shouted at or being & strongly disagree & 1 & 3.0 & 1 & 2.7 & 1 & 3.3 & 7 & 38.9 & 10 & 8.5 & \\
\hline $\begin{array}{l}\text { the target of spontaneous } \\
\text { anger (or rage) }\end{array}$ & disagree; & 0 & 0.0 & 1 & 2.7 & 2 & 6.7 & 2 & 11.1 & 5 & 4.2 & $50.01^{* *}$ \\
\hline & Neutral & 6 & 18.2 & 15 & 40.5 & 7 & 23.3 & 2 & 11.1 & 30 & 25.4 & \\
\hline & Agree & 26 & 78.8 & 15 & 40.5 & 18 & 60.0 & 3 & 16.7 & 62 & 52.5 & \\
\hline & strongly agree & 0 & 0.0 & 5 & 13.5 & 2 & 6.7 & 4 & 22.2 & 11 & 9.3 & \\
\hline Intimidating behavior & strongly disagree & 2 & 6.1 & 5 & 13.5 & 0 & 0.0 & 0 & 0.0 & 7 & 5.9 & \\
\hline such as finger-pointing, & disagree; & 4 & 12.1 & 3 & 8.1 & 4 & 13.3 & 0 & 0.0 & 11 & 9.3 & $43.57^{* *}$ \\
\hline space, shoving, & Neutral & 16 & 48.5 & 6 & 16.2 & 15 & 50.0 & 4 & 22.2 & 41 & 34.7 & \\
\hline blocking/barring the way & Agree & 11 & 33.3 & 23 & 62.2 & 6 & 20.0 & 14 & 77.8 & 54 & 45.8 & \\
\hline & strongly agree & 0 & 0.0 & 0 & 0.0 & 5 & 16.7 & 0 & 0.0 & 5 & 4.2 & \\
\hline Hints or signals from & strongly disagree & 19 & $57.6 \%$ & 13 & $35.1 \%$ & 12 & $40.0 \%$ & 7 & $38.9 \%$ & 51 & $43.2 \%$ & \\
\hline $\begin{array}{l}\text { others that you should } \\
\text { quit your job }\end{array}$ & disagree; & 14 & $42.4 \%$ & 24 & $64.9 \%$ & 18 & $60.0 \%$ & 11 & $61.1 \%$ & 67 & $56.8 \%$ & $4.02^{\mathrm{ns}}$ \\
\hline & strongly disagree & 0 & 0.0 & 3 & 8.1 & 1 & 3.3 & 1 & 5.6 & 5 & $4.2 \%$ & \\
\hline Reneated reminders of & disagree; & 2 & 6.1 & 3 & 8.1 & 1 & 3.3 & 5 & 27.8 & 11 & 9.3 & $38.26^{* *}$ \\
\hline your errors or mistakes & Neutral & 13 & 39.4 & 18 & 48.6 & 4 & 13.3 & 4 & 22.2 & 39 & 33.1 & \\
\hline & Agree & 11 & 33.3 & 10 & 27.0 & 24 & 80.0 & 6 & 33.3 & 51 & 43.2 & \\
\hline & strongly agree & 7 & 21.2 & 3 & 8.1 & 0 & 0.0 & 2 & 11.1 & 12 & 10.2 & \\
\hline Being ignored or facing & strongly disagree & 7 & 21.2 & 6 & 16.2 & 2 & 6.7 & 5 & 27.8 & 20 & 16.9 & \\
\hline $\begin{array}{l}\text { hostile reaction when you } \\
\text { approach. }\end{array}$ & disagree; & 9 & 27.3 & 10 & 27.0 & 8 & 26.7 & 7 & 38.9 & 34 & 28.8 & $14.15^{\mathrm{ns}}$ \\
\hline & Neutral & 14 & 42.4 & 11 & 29.7 & 12 & 40.0 & 6 & 33.3 & 43 & 36.4 & \\
\hline & Agree & 2 & 6.1 & 9 & 24.3 & 6 & 20.0 & 0 & 0.0 & 17 & 14.4 & \\
\hline & strongly agree & 1 & 3.0 & 1 & 2.7 & 2 & 6.7 & 0 & 0.0 & 4 & 3.4 & \\
\hline Persistent criticism of & strongly disagree & 4 & 12.1 & 5 & 13.5 & 2 & 6.7 & 2 & 11.1 & 13 & 11.0 & \\
\hline your work and effort. & disagree; & 7 & 21.2 & 15 & 40.5 & 12 & 40.0 & 9 & 50.0 & 43 & 36.4 & $17.08^{\mathrm{ns}}$ \\
\hline & Neutral & 21 & 63.6 & 14 & 37.8 & 15 & 50.0 & 3 & 16.7 & 53 & 44.9 & \\
\hline & Agree & 1 & 3.0 & 3 & 8.1 & 1 & 3.3 & 4 & 22.2 & 9 & 7.6 & \\
\hline Having your opinions and & strongly disagree & 3 & 9.4 & 2 & 5.4 & 1 & 3.3 & 0 & 0.0 & 6 & 5.1 & \\
\hline & disagree; & 2 & 6.2 & 2 & 5.4 & 3 & 10.0 & 0 & 0.0 & 7 & 6.0 & $12.95^{\mathrm{ns}}$ \\
\hline & Neutral & 16 & 50.0 & 23 & 62.2 & 18 & 60.0 & 17 & 94.4 & 74 & 63.2 & \\
\hline & Agree & 9 & 28.1 & 8 & 21.6 & 5 & 16.7 & 1 & 5.6 & 23 & 19.7 & \\
\hline & strongly agree & 2 & 6.2 & 2 & 5.4 & 3 & 10.0 & 0 & 0.0 & 7 & 6.0 & \\
\hline Practical jokes carried out & strongly disagree & 19 & 57.6 & 12 & 32.4 & 5 & 16.7 & 2 & 11.1 & 38 & 32.2 & \\
\hline $\begin{array}{l}\text { by people you don't get } \\
\text { on with. }\end{array}$ & disagree; & 8 & 24.2 & 16 & 43.2 & 13 & 43.3 & 2 & 11.1 & 39 & 33.1 & $40.27^{* *}$ \\
\hline & Neutral & 4 & 12.1 & 5 & 13.5 & 12 & 40.0 & 13 & 72.2 & 34 & 28.8 & \\
\hline & Agree & 1 & 3.0 & 3 & 8.1 & 0 & 0.0 & 1 & 5.6 & 5 & 4.2 & \\
\hline & strongly agree & 1 & 3.0 & 1 & 2.7 & 0 & 0.0 & 0 & 0.0 & 2 & 1.7 & \\
\hline
\end{tabular}




\begin{tabular}{|c|c|c|c|c|c|c|c|c|c|c|c|c|}
\hline \multirow[t]{3}{*}{ Items } & & \multicolumn{8}{|c|}{ units } & \multirow{2}{*}{\multicolumn{2}{|c|}{$\begin{array}{c}\text { Total } \\
(\mathrm{n}=118)\end{array}$}} & \multirow[b]{3}{*}{$\mathbf{X}^{2}$} \\
\hline & & \multicolumn{2}{|c|}{$\begin{array}{l}\text { Hemodialysis } \\
\quad(n=33)\end{array}$} & \multicolumn{2}{|c|}{$\begin{array}{c}\text { Preterm } \\
(n=37)\end{array}$} & \multicolumn{2}{|c|}{$\begin{array}{l}\text { PICU } \\
(n=30)\end{array}$} & \multicolumn{2}{|c|}{$\begin{array}{c}\begin{array}{c}\text { Pediatric } \\
\text { department } \\
(\mathrm{n}=18)\end{array} \\
.\end{array}$} & & & \\
\hline & & No & $\%$ & No & $\%$ & No & $\%$ & No & $\%$ & No & $\%$ & \\
\hline \multirow{5}{*}{$\begin{array}{lll}\text { Being given tasks } & \text { of } \\
\text { unreasonable } & \text { or } \\
\text { impossible targets } & \text { or } \\
\text { deadlines. } & & \end{array}$} & strongly disagree & 8 & 24.2 & 11 & 29.7 & 5 & 16.7 & 0 & 0.0 & 24 & 20.3 & \multirow{5}{*}{$21.88^{\mathrm{ns}}$} \\
\hline & disagree; & 15 & 45.5 & 18 & 48.6 & 10 & 33.3 & 15 & 83.3 & 58 & 49.2 & \\
\hline & Neutral & 8 & 24.2 & 6 & 16.2 & 10 & 33.3 & 3 & 16.7 & 27 & 22.9 & \\
\hline & Agree & 2 & 6.1 & 2 & 5.4 & 3 & 10.0 & 0 & 0.0 & 7 & 5.9 & \\
\hline & strongly agree & 0 & 0.0 & 0 & 0.0 & 2 & 6.7 & 0 & 0.0 & 2 & 1.7 & \\
\hline \multirow{5}{*}{$\begin{array}{l}\text { Having allegations made } \\
\text { against you. }\end{array}$} & strongly disagree & 17 & 51.5 & 10 & 27.0 & 4 & 13.3 & 3 & 16.7 & 34 & 28.8 & \multirow{5}{*}{$22.52^{\mathrm{n}}$} \\
\hline & disagree; & 12 & 36.4 & 14 & 37.8 & 15 & 50.0 & 10 & 55.6 & 51 & 43.2 & \\
\hline & Neutral & 4 & 12.1 & 7 & 18.9 & 9 & 30.0 & 4 & 22.2 & 24 & 20.3 & \\
\hline & Agree & 0 & 0.0 & 3 & 8.1 & 2 & 6.7 & 1 & 5.6 & 6 & 5.1 & \\
\hline & strongly agree & 0 & 0.0 & 3 & 8.1 & 0 & 0.0 & 0 & 0.0 & 3 & 2.5 & \\
\hline \multirow{5}{*}{$\begin{array}{l}\text { Excessive monitoring of } \\
\text { your work. }\end{array}$} & strongly disagree & 3 & 9.1 & 0 & 0.0 & 2 & 6.7 & 3 & 16.7 & 8 & 6.8 & \multirow{5}{*}{$42.39^{* *}$} \\
\hline & disagree; & 3 & 9.1 & 2 & 5.4 & 8 & 26.7 & 8 & 44.4 & 21 & 17.8 & \\
\hline & Neutral & 26 & 78.8 & 20 & 54.1 & 13 & 43.3 & 7 & 38.9 & 66 & 55.9 & \\
\hline & Agree & 0 & 0.0 & 13 & 35.1 & 7 & 23.3 & 0 & 0.0 & 20 & 16.9 & \\
\hline & strongly agree & 1 & 3.0 & 2 & 5.4 & 0 & 0.0 & 0 & 0.0 & 3 & 2.5 & \\
\hline \multirow{5}{*}{$\begin{array}{l}\text { Pressure not to claim } \\
\text { something which by right } \\
\text { you are entitled (e.g. } \\
\text { sick). }\end{array}$} & strongly disagree & 2 & 6.1 & 1 & 2.7 & 0 & .0 & 1 & $5.6 \%$ & 4 & $3.4 \%$ & \multirow{5}{*}{$16.70^{\mathrm{ns}}$} \\
\hline & disagree; & 2 & 6.1 & 2 & 5.4 & 7 & 23.3 & 1 & $5.6 \%$ & 12 & $10.2 \%$ & \\
\hline & Neutral & 12 & 36.4 & 18 & 48.6 & 8 & 26.7 & 9 & $50.0 \%$ & 47 & $39.8 \%$ & \\
\hline & Agree & 16 & $48.5 \%$ & 13 & 35.1 & 15 & 50.0 & 7 & $38.9 \%$ & 51 & $43.2 \%$ & \\
\hline & strongly agree & 1 & $3.0 \%$ & 3 & .1 & 0 & $0.0 \%$ & 0 & $0.0 \%$ & 4 & $3.4 \%$ & \\
\hline \multirow{5}{*}{$\begin{array}{l}\text { Threats of violence or } \\
\text { physical abuse or actual } \\
\text { abuse. }\end{array}$} & strongly disagree & 0 & 0.0 & 1 & 2.7 & 0 & .0 & 0 & $0.0 \%$ & 1 & 0.8 & \multirow{5}{*}{$14.09^{\mathrm{ns}}$} \\
\hline & disagree; & 1 & 3.0 & 2 & 5.4 & 2 & 6.7 & 1 & 5.6 & 6 & 5.1 & \\
\hline & Neutral & 25 & 75.8 & 21 & 56.8 & 13 & 43.3 & 10 & 55.6 & 69 & 58.5 & \\
\hline & Agree & 7 & 21.2 & 11 & 29.7 & 15 & 50.0 & 7 & 38.9 & 40 & 33.9 & \\
\hline & strongly agree & 0 & 0.0 & 2 & 5.4 & 0 & 0.0 & 0 & 0.0 & 2 & 1.7 & \\
\hline \multirow{5}{*}{$\begin{array}{l}\text { Being the subject of } \\
\text { excessive teasing and } \\
\text { sarcasm. }\end{array}$} & strongly disagree & 3 & 9.1 & 0 & 0.0 & 2 & 6.7 & 3 & 16.7 & 8 & 6.8 & \multirow{5}{*}{$42.39^{* *}$} \\
\hline & disagree; & 3 & 9.1 & 2 & 5.4 & 8 & 26.7 & 8 & 44.4 & 21 & 17.8 & \\
\hline & Neutral & 26 & 78.8 & 20 & 54.1 & 13 & 43.3 & 7 & 38.9 & 66 & 55.9 & \\
\hline & Agree & 0 & 0.0 & 13 & 35.1 & 7 & 23.3 & 0 & 0.0 & 20 & 16.9 & \\
\hline & strongly agree & 1 & 3.0 & 2 & 5.4 & 0 & 0.0 & 0 & 0.0 & 3 & 2.5 & \\
\hline \multirow[t]{4}{*}{$\begin{array}{l}\text { Being exposed to an } \\
\text { unmanageable workload }\end{array}$} & disagree & 9 & 27.3 & 10 & 27.0 & 0 & 0.0 & 0 & 0.0 & 19 & 16.1 & \multirow{4}{*}{$52.42^{* *}$} \\
\hline & Neutral & 15 & 45.5 & 20 & 54.1 & 4 & 13.3 & 3 & 16.7 & 42 & 35.6 & \\
\hline & Agree & 9 & 27.3 & 7 & 18.9 & 18 & 60.0 & 10 & 55.6 & 44 & 37.3 & \\
\hline & strongly agree & 0 & 0.0 & 0 & 0.0 & 8 & 26.7 & 5 & 27.8 & 13 & 11.0 & \\
\hline
\end{tabular}

\footnotetext{
NB: ${ }^{\text {ns }}$ non statistically significant at $\mathrm{P}>0.05$
}

highly statistical significant at $\mathrm{P}<0.001$

Table 2 clarifies the percentage of exposure to workplace bullying behaviors among studied staff nurses in different studied areas $(n=118)$. It can be seen from this table that, there was a highly statistical significant difference among the studied nurses regarding exposure to the following workplace bullying behaviors (someone withholding information which affects your performance, being ordered to do work below your level of competence, having key areas of responsibility removed or replace with more trivial or unpleasant tasks, Spreading of gossip and rumours about you, being shouted at or being the target of spontaneous anger (or rage), intimidating behavior such as finger-pointing, invasion of personal space, shoving, blocking/barring the way, repeated reminders of your errors or mistakes, practical jokes carried out by people you don't get on with, excessive monitoring of your work, being the subject of excessive teasing and sarcasm, and being exposed to an unmanageable workload) as $(\mathrm{p}<0.001)$. 
Table (3): Agreement of nurses regarding children's exposure to some adverse event (n=118)

\begin{tabular}{|c|c|c|c|c|c|c|c|c|c|c|c|c|}
\hline \multirow{3}{*}{ Adverse Events } & & \multicolumn{8}{|c|}{ units } & \multirow{2}{*}{\multicolumn{2}{|c|}{$\begin{array}{l}\text { Total } \\
(n=118)\end{array}$}} & \multirow[t]{3}{*}{$\mathrm{X}^{2}$} \\
\hline & & \multicolumn{2}{|c|}{$\begin{array}{l}\text { Hemodialysis } \\
(\mathrm{n}=33)\end{array}$} & \multicolumn{2}{|c|}{$\begin{array}{l}\text { NICU } \\
(n=37)\end{array}$} & \multicolumn{2}{|c|}{$\begin{array}{l}\text { PICU } \\
(\mathrm{n}=30)\end{array}$} & \multicolumn{2}{|c|}{$\begin{array}{l}\text { Pediatric } \\
\text { department } \\
(n=18)\end{array}$} & & & \\
\hline & & No & $\%$ & No & $\%$ & No & $\%$ & No & $\%$ & No & $\%$ & \\
\hline \multirow{4}{*}{$\begin{array}{l}\text { Children received wrong } \\
\text { medication or dose }\end{array}$} & strongly disagree & 7 & 21.2 & 6 & 16.2 & 5 & 16.7 & 2 & 11.1 & 20 & 16.9 & \multirow{4}{*}{$11.12^{\mathrm{ns}}$} \\
\hline & disagree; & 6 & 18.2 & 17 & 45.9 & 15 & 50.0 & 9 & 50.0 & 47 & 39.8 & \\
\hline & Neutral & 16 & 48.5 & 11 & 29.7 & 8 & 26.7 & 7 & 38.9 & 42 & 35.6 & \\
\hline & Agree & 4 & 12.1 & 3 & 8.1 & 2 & 6.7 & 0 & 0.0 & 9 & 7.6 & \\
\hline \multirow[t]{4}{*}{ Nosocomial infection } & strongly disagree & 7 & 21.2 & 3 & 8.1 & 4 & 13.3 & 0 & .0 & 14 & 11.9 & \multirow{4}{*}{$16.63^{\mathrm{ns}}$} \\
\hline & disagree; & 9 & 27.3 & 15 & 40.5 & 8 & 26.7 & 11 & 61.1 & 43 & 36.4 & \\
\hline & Neutral & 17 & 51.5 & 16 & 43.2 & 13 & 43.3 & 5 & 27.8 & 51 & 43.2 & \\
\hline & Agree & 0 & 0.0 & 3 & 8.1 & 5 & 16.7 & 2 & 11.1 & 10 & 8.5 & \\
\hline \multirow[t]{4}{*}{ Fall } & strongly disagree & 11 & 33.3 & 10 & 27.0 & 0 & 0.0 & 0 & 0.0 & 21 & 17.8 & \multirow[t]{4}{*}{$25.58^{*}$} \\
\hline & disagree; & 6 & 18.2 & 12 & 32.4 & 12 & 40.0 & 4 & 22.2 & 34 & 28.8 & \\
\hline & Neutral & 16 & 48.5 & 11 & 29.7 & 15 & 50.0 & 11 & 61.1 & 53 & 44.9 & \\
\hline & Agree & 0 & 0.0 & 4 & 10.8 & 3 & 10.0 & 3 & 16.7 & 10 & 8.5 & \\
\hline \multirow[t]{4}{*}{ Children fall with injury } & strongly disagree & 8 & 24.2 & 10 & 27.0 & 9 & 30.0 & 6 & 33.3 & 33 & 28.0 & \multirow[t]{4}{*}{$10.73^{\mathrm{ns}}$} \\
\hline & disagree; & 7 & 21.2 & 10 & 27.0 & 14 & 46.7 & 8 & 44.4 & 39 & 33.1 & \\
\hline & Neutral & 12 & 36.4 & 12 & 32.4 & 5 & 16.7 & 2 & 11.1 & 31 & 26.3 & \\
\hline & Agree & 6 & 18.2 & 5 & 13.5 & 2 & 6.7 & 2 & 11.1 & 15 & 12.7 & \\
\hline \multirow{4}{*}{$\begin{array}{l}\text { Complains from patients } \\
\text { and family }\end{array}$} & strongly disagree & 10 & 30.3 & 8 & 21.6 & 7 & 23.3 & 3 & 16.7 & 28 & 23.7 & \multirow[t]{4}{*}{$10.61^{\mathrm{ns}}$} \\
\hline & disagree; & 6 & 18.2 & 4 & 10.8 & 6 & 20.0 & 1 & 5.6 & 17 & 14.4 & \\
\hline & Neutral & 13 & 39.4 & 18 & 48.6 & 13 & 43.3 & 14 & 77.8 & 58 & 49.2 & \\
\hline & Agree & 4 & 12.1 & 7 & 18.9 & 4 & 13.3 & 0 & $0.0 \%$ & 15 & 12.7 & \\
\hline
\end{tabular}

\footnotetext{
* Statistically significant at $\mathrm{P}<0.05{ }^{\mathrm{ns}}$ non statistically significant at $\mathrm{P}>0.05$
}

Table 3 shows Agreement of nurses regarding children's exposure to some adverse event. It was illustrated that the studied nurses' in hemodialysis unit were neutrally agree about that children can exposure to all aspects of children adverse events as a result of their exposure to workplace bullying behavior. While, in NICU the highest percentage of staff nurses were neutrally agree that children can exposure to only three aspects related to children adverse events which include (nosocomial infection, 43.2\%; children fall with injury, 32.4\%; and complains from patients and family, $48.6 \%$ ) as a result from of their exposure to workplace bullying behavior.

Also, it was found that, PICU nurses were neutrally agree on adverse events that children can possess as of staff nurses exposure to different workplace bullying behaviors were nosocomial infection, fall and complains from patients and family $(43.3 \%, 50.0 \%, 43.3 \%)$. Meanwhile, the highest percentage of staff nurses on pediatric department were neutrally agree that the most common of adverse events that children can exposure to it related to their exposure to workplace bullying behaviors was complains from patients and family (77.8\%). Farther more there was a statistical significant difference among studied nurses only regarding children fall as $(\mathrm{p}<0.05)$. 
Table (4): Mean of frequencies for nurses exposure to bullying behaviors and children's exposure to some adverse events in different studied areas

\begin{tabular}{|c|c|c|c|c|c|}
\hline \multirow[t]{3}{*}{ Items } & Unit & & & & \multirow{3}{*}{ Anova test } \\
\hline & $\begin{array}{l}\text { Hemodialysis } \\
(\mathrm{n}=33)\end{array}$ & $\begin{array}{l}\text { Preterm } \\
(n=37)\end{array}$ & $\begin{array}{l}\text { PICU } \\
(n=30)\end{array}$ & $\begin{array}{l}\text { Pediatric } \\
\text { department } \\
(n=18)\end{array}$ & \\
\hline & $\overline{\mathbf{x}} \pm \mathbf{S D}$ & $\overline{\mathbf{x}} \pm \mathbf{S D}$ & $\overline{\mathbf{x}} \pm \mathbf{S D}$ & $\overline{\mathbf{x}} \pm \mathbf{S D}$ & \\
\hline $\begin{array}{l}\text { Nurses exposure to } \\
\text { bullying behavior's }\end{array}$ & $67.52 \pm 5.86$ & $69.05 \pm 6.35$ & $70.0 \pm 6.57$ & $68.00 \pm 6.98$ & $.730^{\mathrm{ns}}$ \\
\hline $\begin{array}{l}\text { children's exposure to } \\
\text { adverse events }\end{array}$ & $11.73 \pm 2.98$ & $12.05 \pm 2.47$ & $12.77 \pm 3.29$ & $12.39 \pm 2.66$ & $.463^{\mathrm{ns}}$ \\
\hline
\end{tabular}

${ }^{\mathrm{ns}}$ non statistically significant at $\mathrm{P}>0.05$

Table 4 clarified mean total scores of nurses' exposure to bullying behaviors and children's exposure to some adverse events in different studied areas. As shown from the table premature intensive care (PICU) unit was the highest mean total score for both; nurses' exposure to workplace bullying behavior and children's exposure to some adverse events among different study areas. Also, it was observed that there was no statistical significant difference between studied nurses that exposure bullying behavior and children's exposure to some adverse events in different study areas as, $(\mathrm{P}>0.05)$.

Table (5): Level of nurses' exposure to bullying behaviors and children's exposure to some adverse events in different studied areas

\begin{tabular}{|c|c|c|c|c|c|c|c|c|c|c|c|}
\hline \multirow[t]{3}{*}{ Items } & \multicolumn{8}{|c|}{ Unit } & \multirow{2}{*}{\multicolumn{2}{|c|}{$\begin{array}{l}\text { Total } \\
(n=118)\end{array}$}} & \multirow[t]{3}{*}{$X$} \\
\hline & \multicolumn{2}{|c|}{$\begin{array}{l}\text { Hemodialysis } \\
(\mathrm{n}=33)\end{array}$} & \multicolumn{2}{|c|}{$\begin{array}{l}\text { Preterm } \\
(n=37)\end{array}$} & \multicolumn{2}{|c|}{$\begin{array}{l}\text { PICU } \\
(n=30)\end{array}$} & \multicolumn{2}{|c|}{$\begin{array}{l}\text { Pediatric } \\
\text { department } \\
(n=18)\end{array}$} & & & \\
\hline & No & $\%$ & No & $\%$ & No & $\%$ & No & $\%$ & No & $\%$ & \\
\hline \multicolumn{11}{|c|}{$\begin{array}{l}\text { Level of nurses' exposure } \\
\text { to bullying behaviors }\end{array}$} & \multirow{4}{*}{$4.55^{\mathrm{ns}}$} \\
\hline Low & 7 & 21.2 & 13 & 35.1 & 9 & 30.0 & 4 & 22.2 & 33 & 28.0 & \\
\hline Moderate & 24 & 72.7 & 21 & 56.8 & 15 & 50.0 & 12 & 66.7 & 72 & 61.0 & \\
\hline High & 2 & 6.1 & 3 & 8.1 & 6 & 20.0 & 2 & 11.1 & 13 & 11.0 & \\
\hline \multicolumn{11}{|c|}{$\begin{array}{l}\text { Level of } \quad \text { children's } \\
\text { exposure to some adverse } \\
\text { events }\end{array}$} & \multirow{4}{*}{$9.61^{\mathrm{ns}}$} \\
\hline Low & 8 & 24.2 & 8 & 21.6 & 6 & 20.0 & 3 & 16.7 & 25 & 21.2 & \\
\hline Moderate & 20 & 60.6 & 23 & 62.2 & 18 & 60.0 & 12 & 66.6 & 73 & 61.9 & \\
\hline High & 5 & 15.2 & 6 & 16.2 & 6 & 20.0 & 3 & 16.7 & 20 & 16.9 & \\
\hline
\end{tabular}

${ }^{\mathrm{ns}}$ non statistically significant at $\mathrm{P}>0.05$

Table (5): Level of nurses' exposure to bullying behaviors and children's exposure to adverse events in different studied areas. It was obvious that, the highest percentage of the studied nurses in different studied areas had moderate level of workplace bullying behavior's exposure $(61.0 \%)$ and children had moderate level of exposure to some adverse events $(61.9 \%)$ as reported by the studied staff nurses' and there were no statistical significant differences founded $(\mathrm{P}>0.05)$. 


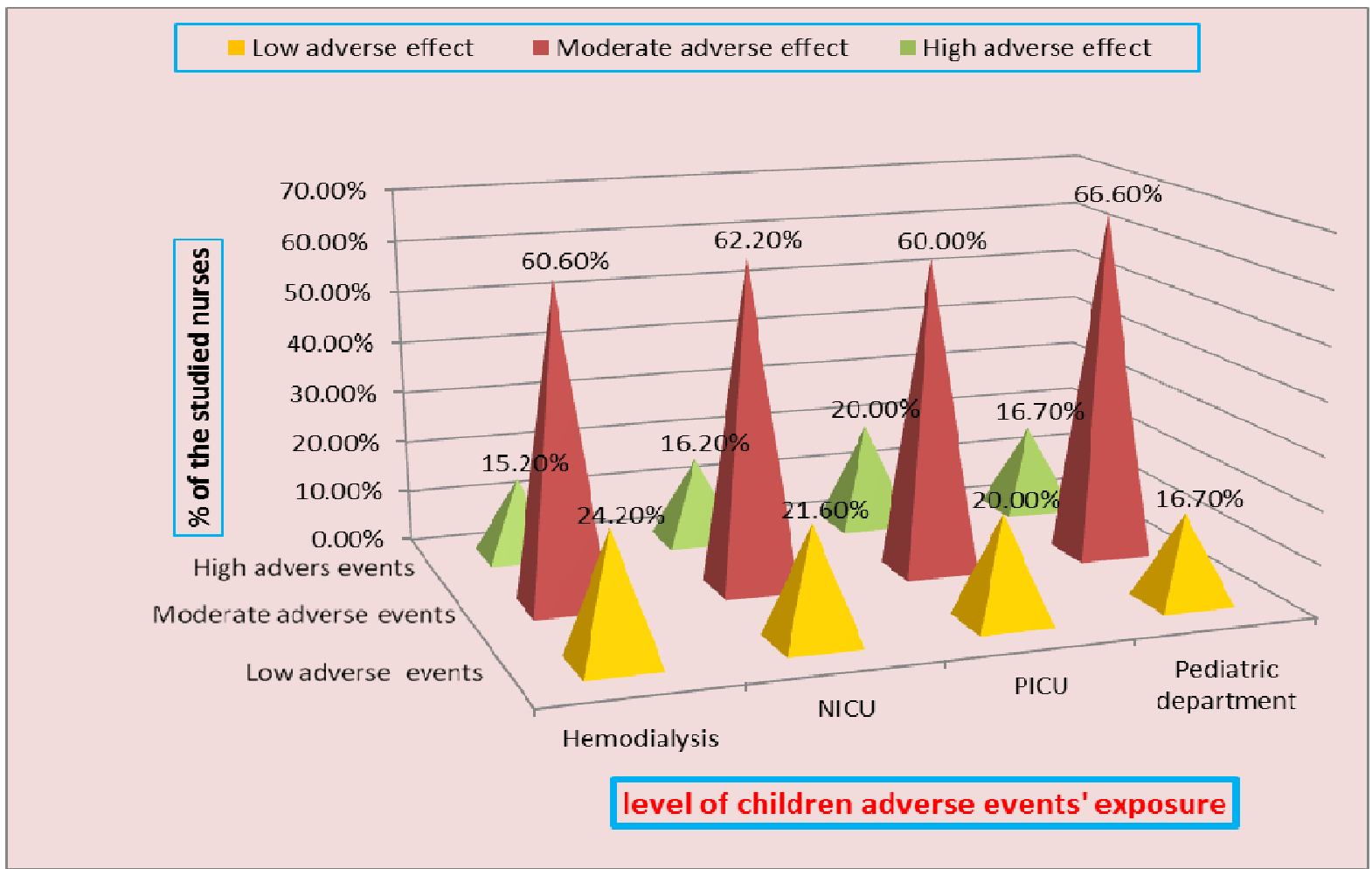

\section{Figure (2): level of children's exposure to adverse events at different of studies areas.}

Figure 2 shows the level of children's exposure to adverse events at different of studies areas. It was obvious that children in PICU had the highest exposure to adverse event (20.0\%) as reported by staff nurses. On contrast, children in hemodialysis unit had the lowest exposure level to adverse events $(24.2 \%)$ as reported by staff nurses. Furthermore, children in pediatrics department were moderately exposure level to adverse events $(66.6 \%)$.

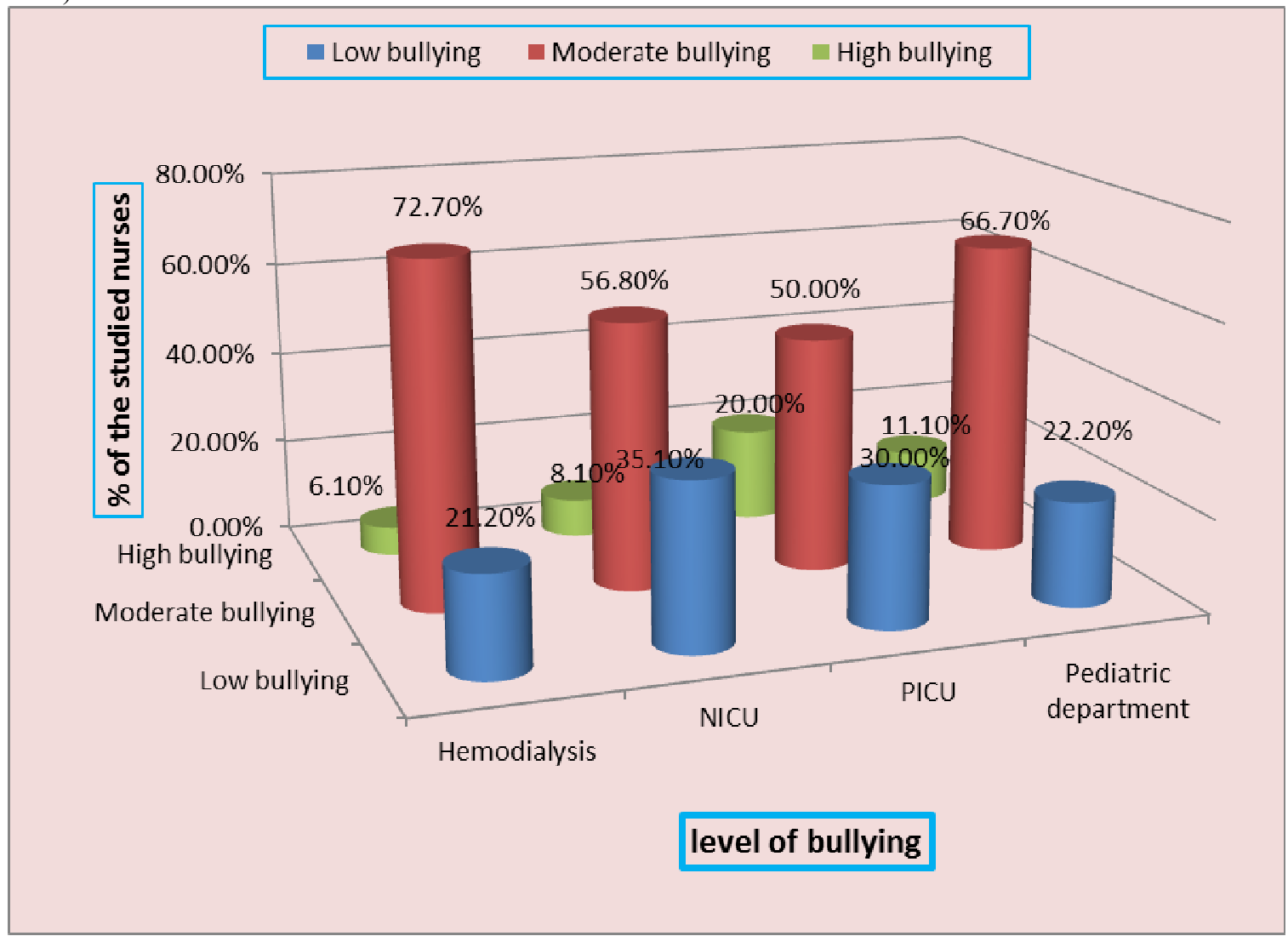

Figure (3): level of bullying behaviors among studied nurses' in studied areas 
Figure 3 shows the level of bullying behaviors among studied nurses' in studied areas. As shown nurses in heamodialysis had the moderately exposure levels to workplace bullying behaviors $(72.7 \%)$ while, nurses in NICU had the lowest exposure level to workplace bullying behaviors (35.1\%). Farthermore nurses in PICU had the highest exposure level to workplace bullying behaviors $(22.0 \%)$.

Table (6): Distribution of satisfaction scale for the studied parents in different studied areas of the care provided for their children $(n=100)$

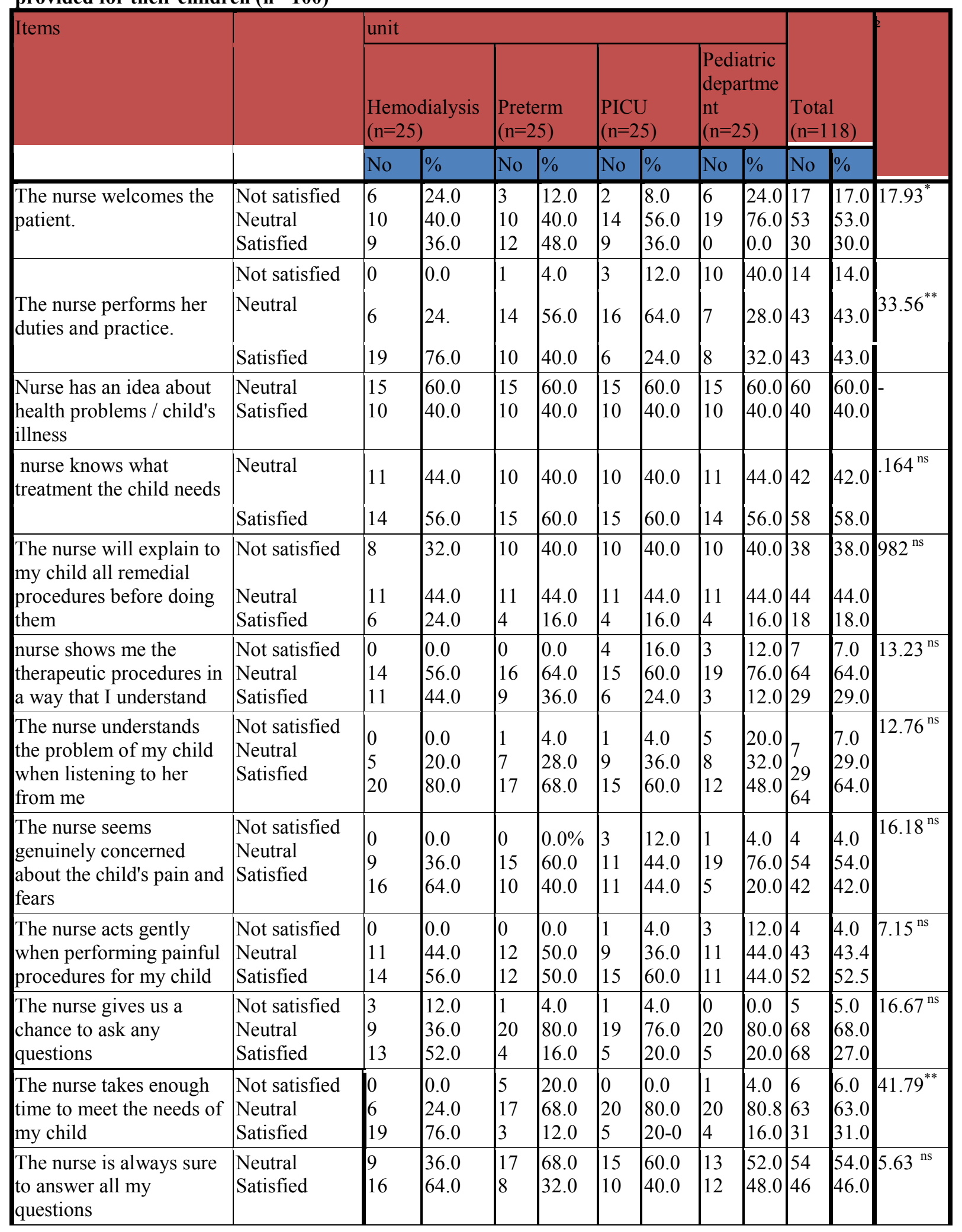




\begin{tabular}{|c|c|c|c|c|c|c|c|c|c|c|c|c|}
\hline \multirow[t]{3}{*}{ Items } & & \multicolumn{8}{|c|}{ unit } & \multirow{2}{*}{\multicolumn{2}{|c|}{$\begin{array}{l}\text { Total } \\
(n=118)\end{array}$}} & \\
\hline & & \multicolumn{2}{|c|}{$\begin{array}{l}\text { Hemodialysis } \\
(\mathrm{n}=25)\end{array}$} & \multicolumn{2}{|c|}{$\begin{array}{l}\text { Preterm } \\
(n=25)\end{array}$} & \multicolumn{2}{|c|}{$\begin{array}{l}\text { PICU } \\
(\mathrm{n}=25)\end{array}$} & \multicolumn{2}{|c|}{$\begin{array}{l}\text { Pediatric } \\
\text { departme } \\
\mathrm{nt} \\
(\mathrm{n}=25)\end{array}$} & & & \\
\hline & & No & $\%$ & No & $\%$ & No & $\%$ & No & $\%$ & No & $\%$ & \\
\hline $\begin{array}{l}\text { The nurse is given } \\
\text { instructions on how to } \\
\text { take care of my child at } \\
\text { home }\end{array}$ & $\begin{array}{l}\text { Neutral } \\
\text { Satisfied }\end{array}$ & $\begin{array}{l}11 \\
14\end{array}$ & $\begin{array}{l}44.0 \\
56.0\end{array}$ & $\begin{array}{l}16 \\
9\end{array}$ & $\begin{array}{l}64.0 \\
36.0\end{array}$ & $\begin{array}{l}17 \\
8\end{array}$ & $\begin{array}{l}68.0 \\
32.0\end{array}$ & $\begin{array}{l}19 \\
6\end{array}$ & $\begin{array}{l}76.0 \\
24.0\end{array}$ & $\begin{array}{l}63 \\
37\end{array}$ & $\begin{array}{l}63.0 \\
37.0\end{array}$ & $5.96^{\mathrm{ns}}$ \\
\hline $\begin{array}{l}\text { The nurse tells me what } \\
\text { to expect for my child at } \\
\text { home }\end{array}$ & $\begin{array}{l}\text { Neutral } \\
\text { Satisfied }\end{array}$ & $\begin{array}{l}15 \\
10\end{array}$ & $\begin{array}{l}60.0 \\
40.0\end{array}$ & 20 & $\begin{array}{l}80.0 \% \\
20.0 \%\end{array}$ & 32 & $\begin{array}{l}88.0 \\
12.0\end{array}$ & 4 & $\begin{array}{l}84.0 \\
16.0\end{array}$ & 22 & $\begin{array}{l}78.0 \\
22.0\end{array}$ & $6.76^{\mathrm{ns}}$ \\
\hline
\end{tabular}

${ }^{\text {ns }}$ non statistically significant at $\mathrm{P}>0.05 *$ statistically significant at $\mathrm{P}<0.05{ }^{* *}$ highly statistical significant at $\mathrm{P}<0.001$

Table 6 presents studied parents satisfaction in different studied areas of the care provided for their children. It can be seen from this table that statistical significant differences were found at $5 \%$ and $1 \%$ between parent's satisfaction in different studied areas about the nurse welcomes the patient, the nurse performs her duties and practice and the nurse takes enough time to meet the needs of the child respectively.

Table (7): Level of total scores of parent's satisfaction in different areas of the care provided for their children $(\mathbf{n}=\mathbf{1 0 0})$

\begin{tabular}{|c|c|c|c|c|c|c|c|c|c|c|c|}
\hline \multirow[t]{3}{*}{ Items } & \multicolumn{8}{|c|}{ Unit } & \multirow{2}{*}{\multicolumn{2}{|c|}{$\begin{array}{l}\text { Total } \\
(\mathrm{n}=118)\end{array}$}} & \multirow[t]{3}{*}{$\mathrm{X}$} \\
\hline & \multicolumn{2}{|c|}{$\begin{array}{l}\text { Hemodialysis } \\
(\mathrm{n}=25)\end{array}$} & \multicolumn{2}{|c|}{$\begin{array}{l}\text { Preterm } \\
(\mathrm{n}=25)\end{array}$} & \multicolumn{2}{|c|}{$\begin{array}{l}\text { PICU } \\
(n=25)\end{array}$} & \multicolumn{2}{|c|}{$\begin{array}{l}\text { Pediatric } \\
\text { department } \\
(n=25)\end{array}$} & & & \\
\hline & No & $\%$ & No & $\%$ & No & $\%$ & No & $\%$ & No & $\%$ & \\
\hline \multicolumn{12}{|l|}{ Level of satisfaction } \\
\hline Low satisfaction $(<17)$ & 5 & 20.0 & 10 & 40.0 & 13 & 52.0 & 15 & 60.0 & 43 & 43.0 & \\
\hline Moderate satisfaction (18-21) & 11 & 44.0 & 15 & 60.0 & 11 & 44.0 & 8 & 32.0 & 45 & 45.0 & $24.14^{* *}$ \\
\hline High Satisfaction $(>21)$ & 9 & 36.0 & 0 & 0.0 & 1 & 4.0 & 2 & 8.0 & 12 & 12.0 & \\
\hline
\end{tabular}

highly statistical significant at $\mathrm{P}<0.001$

Table 7 shows that level of total scores of parent's satisfaction in different areas of the care provided for their children. It was clear that, the highest percentage of the studied parents have moderate level of satisfaction of nursing care that was provided to their children (45\%). On contrast, the lowest percentages of the studied parents have low level of satisfaction of nursing care that was provided to their children $(12 \%)$. Therefore, there were highly statistical significant differences between the studied parents at different study areas $(\mathrm{P}<0.001)$. 


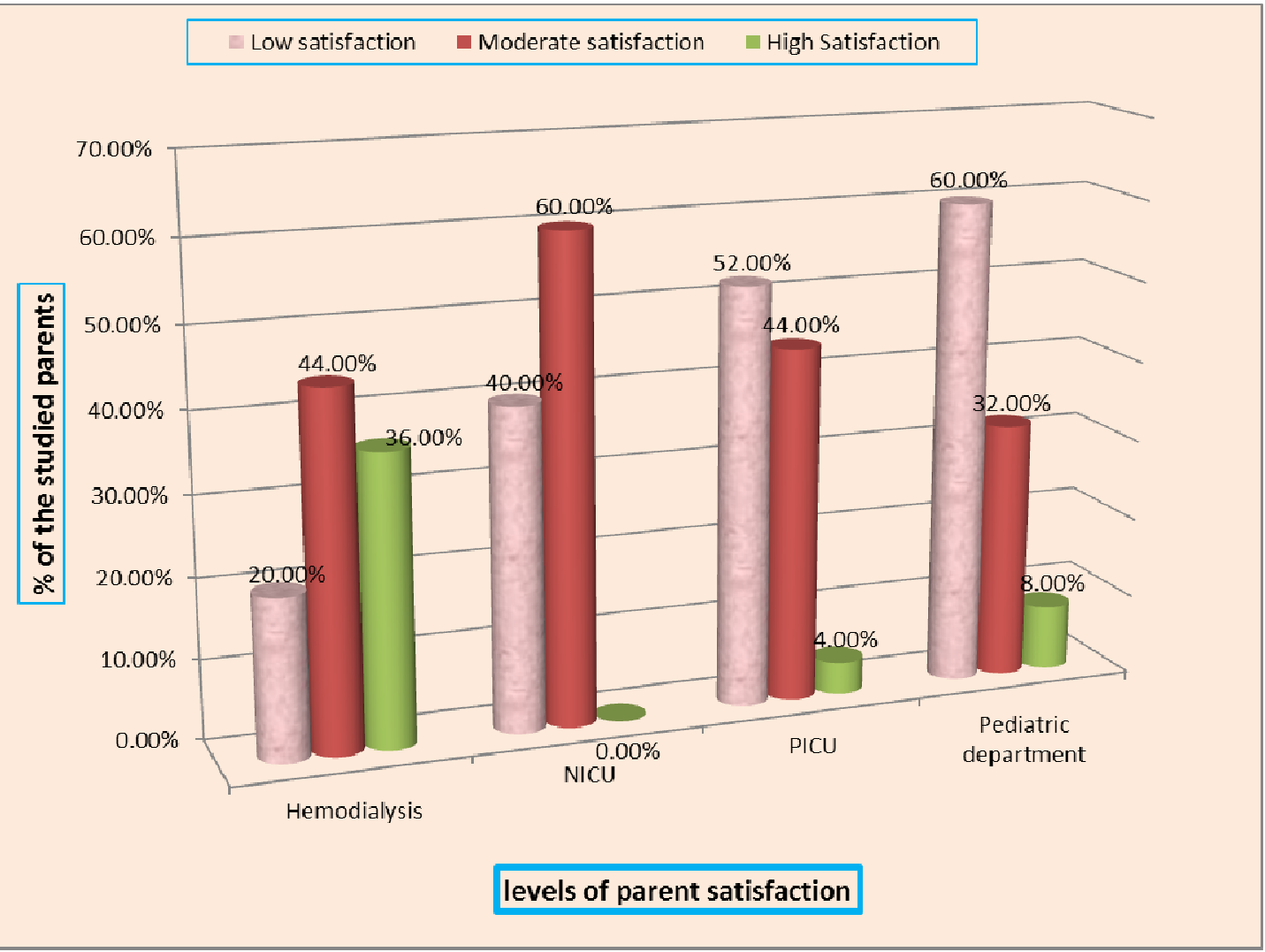

Figure (4): level of parent's satisfaction in different areas of the care provided for their children

Figure 4 reveals that the level of parent's satisfaction in different studied areas of the care provided for their children. It was clear that the lowest level of parents satisfaction was founded in pediatrics department $(60.0 \%)$. while, the highest level of parents satisfaction was found in hemodialysis unit (36\%).

Table (8): Mean total scores of parent's satisfaction in different areas of the care provided for their children

\begin{tabular}{|c|c|c|c|c|c|}
\hline \multirow[t]{3}{*}{ Items } & \multicolumn{4}{|l|}{ Unit } & \multirow{3}{*}{ Anova test } \\
\hline & $\begin{array}{l}\begin{array}{l}\text { Hemodialysis } \\
(\mathrm{n}=25)\end{array} \\
\end{array}$ & $\begin{array}{l}\text { NICU } \\
(n=25)\end{array}$ & $\begin{array}{l}\begin{array}{l}\text { PICU } \\
(n=25)\end{array} \\
(n+10\end{array}$ & $\begin{array}{l}\text { Pediatric department } \\
(\mathrm{n}=25)\end{array}$ & \\
\hline & $\overline{\mathbf{x}} \pm \mathbf{S D}$ & $\overline{\mathbf{x}} \pm \mathbf{S D}$ & $\overline{\mathbf{x}} \pm \mathbf{S D}$ & $\overline{\mathbf{x}} \pm \mathbf{S D}$ & \\
\hline Total parent's satisfaction & $20.96 \pm 2.24$ & $18.24 \pm 1.88$ & $17.88 \pm 1.96$ & $16.36 \pm 3.09$ & $16.676^{* *}$ \\
\hline
\end{tabular}

highly statistical significant at $\mathrm{P}<0.001$

Table 8 shows mean and standard deviation of the total parent's satisfaction of care provided for their children in the in deferent studied areas. It was founded that parents in heamodialysis have the highest mean and standard deviation regarding their satisfaction of the care for that was provided to their children $(20.96 \pm 2.24)$. Therefore, there was a highly statistical significant difference at $1 \%$ level of statistical significance.

Table (9): Correlation between total of nurses exposure to bullying behaviors score and demographic characteristics for their in different studied areas

\begin{tabular}{|l|l|l|}
\hline \multirow{2}{*}{ Items } & \multicolumn{2}{|l|}{ Total score of bullying } \\
\cline { 2 - 4 } & $\mathrm{r}$ & $\mathrm{P}$-value \\
\hline Age & $\mathbf{- . 1 9 4 *}$ & $\mathbf{. 0 3 5}$ \\
\hline Years of experience & $\mathbf{- . 2 5 3 * *}$ & $\mathbf{. 0 0 6}$ \\
\hline Educational qualifications & $\mathbf{- . 1 8 4 *}$ & $\mathbf{. 0 4 5}$ \\
\hline
\end{tabular}

Table 9 shows Correlation between total of nurses exposure to bullying behaviors score and demographic characteristics for their in different studied areas. There was negative statistical significant correlation between total of nurses exposure to bullying behaviors score and age, years of experience and educational qualifications. 
Table (10): Correlation between total score of nurses exposure to bullying behaviors and children exposure to adverse events and parent's satisfaction.

\begin{tabular}{|l|l|l|}
\hline \multirow{2}{*}{ Items } & \multicolumn{2}{|l|}{ Total score of bullying } \\
\cline { 2 - 3 } & $\mathrm{r}$ & $\mathrm{P}$-value \\
\hline Total score of children adverse events & $\mathbf{. 2 1 8 *}$ & $\mathbf{. 0 1 5}$ \\
\hline Total score of parent's satisfaction & $\mathbf{- . 3 2 1 * *}$ & $\mathbf{. 0 0 1}$ \\
\hline
\end{tabular}

Table 10 shows Correlation between total score of nurses exposure to bullying behaviors and children exposure to adverse events and parent's satisfaction. There was positive statistical significant correlation between total score of nurses exposure to bullying behaviors and children exposure to adverse events. While, there was negative statistical significant correlation between total score of nurses exposure to bullying behaviors and parent's satisfaction.

Table (11): Correlation between total score of children exposure to adverse events and parent's satisfaction

\begin{tabular}{|l|l|l|}
\hline \multirow{2}{*}{ Items } & \multicolumn{2}{|l|}{ total score of parent's satisfaction } \\
\cline { 2 - 3 } & $\mathrm{r}$ & $\mathrm{P}$-value \\
\hline total score of adverse effect & $\mathbf{- . 2 2 7 *}$ & $\mathbf{. 0 1 1}$ \\
\hline
\end{tabular}

Table 11 reveals correlation between total score of children exposure to adverse events and parent's satisfaction. There was negative statistical significant correlation between children exposure to adverse events and parent's satisfaction.

\section{Discussion:}

Although bullying behaviors at the workplace are seen in all sector, it has been determined that they are more widespread in healthcare organizations ${ }^{[31]}$. The current study questions were what was the level of workplace bullying among pediatric nurses at selected setting?, What was nurses' agreement regarding children exposure to some of adverse events at selected setting?, What was the level of parent's satisfaction of the nursing care provided to their children at selected setting? And was there a correlation of workplace bullying, children exposure to some of adverse events and parent satisfaction at selected setting?

In relation to question one: The present finding illustrated that, the highest percentage of the studied nurses in different studied areas had moderately level of workplace bullying behavior's exposure. This might be due to the continuous, unfair criticism and attacks on nurses personality and professional status were usually done while in the presence of others and within a group. So, it can causes nurses who are the direct targets of bullying experience negative work behaviors, such as decreased job satisfaction, decreased job performance, decreased productivity, less motivation, increased possibility of making a work-related error, and decreased connectivity to the job and institution.

This finding came in agreement with Y1ldırım, (2009) who conducted a study about "Bullying among nurses and its effects" He mentioned that, Workplace bullying is a assessable problem that negatively affects the psychology and performance of the nurses ${ }^{[32]}$. Moreover, this result was in the same line with Heather \& Laschinger, (2014) who conducted a study about "Impact of Workplace Mistreatment on Patient Safety Risk and Nurse-Assessed Patient Outcomes" They reported that, Bullying and workplace incivility have adverse effects on nurse-assessed patient quality through their effect on perceptions of patient safety risk ${ }^{[33]}$. Also, these findings came in agreement with Meng, (2016) who conducted a research about "Management of work place bullying in hospital: A review of the use of cognitive rehearsal as an alternative management strategy" The researchers clarified that exposure to bullying behaviors such as verbal abuse has been known to have negative impacts on nurses' self-esteem, job satisfaction, morale, patient care, work productivity and professional error rates ${ }^{[34]}$.

In relation to level of bullying behaviors among studied nurses in studied areas, the finding illustrated that nurses on PICU had the highest level of bullying behaviors and that these behaviors which nurses exposure to it were being humiliated or ridiculed in connection with their work, being ordered to do work below their level of competence, having key areas of responsibility removed or replace with more trivial or unpleasant tasks, Being ignored or excluded, being shouted at or being the target of spontaneous anger (or rage), Intimidating behavior such as finger-pointing, invasion of personal space, shoving and blocking/barring the way, repeated reminders of their errors or mistakes, being ignored or facing hostile reaction when their approach, having their opinions and views ignored, being given tasks of unreasonable or impossible targets or deadlines, excessive monitoring of their work, Pressure not to claim something which by right you are entitled (e.g. sick), Threats of violence or physical abuse or actual abuse, excessive teasing and sarcasm and being exposed to an unmanageable workload . This could be excessive workload, decrease experience years young nurses and inadequate staffing had significant effects on nurses' exposure to workplace bullying behaviors in PICU.

This finding came in agreement with Laschinger et al., (2013) who conducted a review about "Workplace incivility and new graduate nurses' mental health: the protective role of resiliency" He mentioned that, young 
employees are often sufferers of direct attacks and negative behaviors from older and more experienced employee ${ }^{[35]}$.

In relation to research question two, the present finding found that children in different studied areas had moderate level of exposure to some adverse events reported by the studied staff nurses'. Also, children in PICU had the highest exposure level to adverse events as reported by staff nurses. This might be due to nurses in PICU had the highest level of exposure to bullying behaviors that causes children in this unit had the highest exposure level to some adverse events. This finding was consistent with Lewis \& Malecha, (2011) who conducted a research about "The impact of workplace incivility on the work environment, manager skill, and productivity" They found that although bullying/incivility rates were low, when present, they were linked with a perceived increase in children adverse events and improper quality of patient care ${ }^{[36]}$. In addition, Chipps et al., (2013) determined that workplace bullying had a direct effect on patient quality and patient safety ${ }^{[37]}$.

In relation to research question three, the present finding cleared that the highest percentage of the studied parents in different studied areas have moderate level of satisfaction of nursing care that was provided to their children This might be due to The increase in the rate of bullying among nurses in different units has led to an increase in the incidence of some negative effects among children, which leads to decrease parents satisfaction for the care provided to their children. Also, in hemodialysis unit, highest level of parents satisfaction was found. It was related to parents had other factors that increase their satisfaction as parents share nurses in their children care. These findings were in the same line with Aiken et al., (2012) who conducted a research about "Patient safety, satisfaction, and quality of hospital care: cross sectional surveys of nurses and patients in 12 countries in Europe and the United States" they reported that, negative interpersonal behaviors and bullying among nurses were associated with perceived threats to patient care quality as a consequence of decreased teamwork and poor morale, which ultimately decreases nurses' ability to provide high-quality patient care and ensuring patients and families satisfaction ${ }^{[38]}$.

In relation to question three, the current finding found that there was negative correlation between total of nurse's exposure to bullying behaviors score and age, years of experience and educational qualifications. This result was consistent with Hutchison \& Jackson, (2013) they conducted a study about "Transformational leadership in nursing: towards a more critical interpretation" they reported that there was negative correlation associated bullying with nurses' age $(\mathrm{P}<0.01)^{[39]}$. On the other hand, this finding were inconsistent with Y1ldirım, (2007) who reported that there were positive correlation between total number of years' experience and nurses educational level and exposure to bullying behaviors $(\mathrm{P}>0.05)^{[40]}$.

Also, this study revealed that there was positive correlation between total score of nurses exposure to bullying behaviors and children exposure to adverse events. This result was consistent with Hutchison \& Jackson, (2013) they conducted a study about "Transformational leadership in nursing: towards a more critical interpretation" They mentioned that a positive relation between of workplace bullying and patient safety outcomes ${ }^{[39]}$

In addition, this finding was consistent with Pai \& Lee, (2011) who conducted a study about "Risk factors for workplace violence in clinical registered nurses in Taiwan" they mentioned that, nurses who had highly bullying exposure also had low performance and low patient safety. Also, when the bullying among nurses was increase, patient's safety risks were also increase ${ }^{[41]}$.

On other hand, the finding of this study clarified that there was negative correlation between total score of nurses exposure to bullying behaviors and parent's satisfaction. This could be due to nurses who had bullying can exposure to more pressure and stress that can causes their performance decrease and become more depression. So, that causes direct effect on quality of care and parents satisfaction level of care that provided for their children.

\section{Conclusion}

Based on the finding of the present study, the following is concluded, there was a positive relationship between the exposure of the nurses to the behavior of bullying and the exposure of children to the adverse events, that the increased exposure of nurses to bullying increases the exposure of children to some side effects. Also, there was a negative correlation between the exposure of nurses to bullying behavior and the level of parents 'satisfaction of care given to their children. That means with increase of nurses exposure to workplace bullying led to parents' had low satisfaction level of care provided to their children.

\section{Recommendations}

1. Identifying behaviors of bullying and raising awareness of bullying behavior in society should be the first step in the solution. Subsequently, in-service training programs on bullying behaviors in all pediatric units should be designed and implemented to improve the knowledge and practices of nurses to control and prevent bullying in the workplace.

2. Head nurse should design an action plan with hospital mangers to 
- Develop appropriate policies and procedures need to be developed and shared with all nurses to prevent the development of bullying behaviors.

- Develop of an adverse event reporting and monitoring system in health care organizations and patient safety culture based on assessing the current status of the safety culture in an organization.

- Provide consistent information sharing with parents concerning their child health status and providing high level of support for child's parents in different health care setting.

3. Future studies should be applied on a larger sample to investigate the effect of a nursing intervention program on reducing workplace bullying between pediatric nurses to ensure the generalizability of results.

\section{References}

[1]. Johnson, S., (2015). Workplace Bullying Prevention: A Critical Discourse Analysis. PMC, Journal of Advanced Nursing, Oct; 71(10): 2384-2392.

[2]. Hodgins, M., MacCurtain, S., and Mannix-McNamara, P. (2014). Workplace bullying and incivility: a systematic review of interventions. International Journal Workplace Health Management; 7: 54-72. Retrieved from https://doi.org/10.1108/IJWHM-08-2013-0030

[3]. Johnson, S. \& Rea, R. (2009). Workplace bullying: Concerns for nurse leaders. The Journal of Nursing Administration, 39, 84-90.

[4]. Purpora, C., Blegen, M.A., and Stotts, N. (2012). Horizontal violence among hospital staff nurses related to oppressed self or oppressed group. Journal Professional on Nursing; 28: 306-314 Retrieved from https://doi.org/10.1016/j.profnurs.2012.01.001

[5]. Murray, J. (2009). Workplace bullying in nursing: A problem that can't be ignored. MEDSURG Nursing, $18(5), 273-276$.

[6]. American Nurses Association. (2016). Bullying in the workplace. Nursing Insider news, Retrieved from http://www.nursingworld.org/EspeciallyForYou/Staff-Nurses/Staff-Nurse-News/ANA-Bullying-in-theWorkplace-Publication.html

[7]. Purdy, M. (2011). Effects of Work Environments on Nursing and Patient Outcomes. Electronic Thesis and Dissertation Repository. 92. Retrieved from https://ir.lib.uwo.ca/etd/92

[8]. Laschinger, H., Wong, C., and Grau, A. (2012). The influence of authentic leadership on newly graduated nurses' experiences of workplace bullying, burnout and retention outcomes: a cross-sectional study. International Journal of Nursing Studies. 49: 282-291. Retrieved from https://doi.org/10.1016/j.ijnurstu.2012.05.012

[9]. An, Y. and Kang, J. (2016). Relationship between organizational culture and workplace bullying among Korean nurses. Asian Nursing Research; 10: 234-239. Retrieved from https://doi.org/10.1016/j.anr.2016.06.004

[10]. Bigony, L., Lipke, T., Lundberg, A., McGraw, C., Pagac, G., and Rogers, A. (2009). Lateral violence in the perioperative setting. AORN Journal; 89, 688-96.

[11]. McGrath JM, Rollins W. (2011). Patient safety in the NICU: a comprehensive review. Journal Perinatal Neonatal Nursing; 25 (2):123-32.

[12]. Read, E. and Laschinger, H.K.S. Correlates of new graduate nurses experiences of workplace mistreatment. Journal of Nursing Administration; 2013; 43: 221-228. Retrieved from https://doi.org/10.1097/NNA.0b013e3182895a90

[13]. Edwards, W. (2005) Patient safety in the neonatal intensive care unit. Clinical Perinatology Journal; 32(1):97-106.

[14]. Stavroudis, T., Shore, A., Morlock, L., Hicks R., Bundy, D., and Miller, M. (2010). NICU medication errors: identifying a risk profile for medication errors in the neonatal intensive care unit. Clinical Perinatology Journal, 30(7):459-68.

[15]. Farzi S, Farzi S, Taheri S, Ehsani M., and Moladoost A. (2017). Perspective of Nurses toward the Patient Safety Culture in Neonatal Intensive Care Units. Iranian Journal of Neonatology;8(4). DOI: 10.22038/ijn.2017.22713.1271

[16]. Grosik, C., Snyder, D., Cleary, G. M., \& Breckenridge, D. M. (2013). Identification of Internal and External Stressors in Parents of Newborns in Intensive Care. The Permanente Journal; , 17 (3), 36-41.

[17]. Mahamood, S., \& Reiter, E. (2011). Generating Affective Natural Language for Parents of Neonatal Infants. Association for Computational Linguistics, 12-21.

[18]. Yokoyama, M., Suzuki, M., Takai, Y., Igarashi, A., Noguchi-Watanabe, M., and Yamamoto-Mitani, N. (2016). Workplace bullying among nurses and their related factors in Japan: a cross-sectional survey. Journal of Clinical Nursing; 25: 2478-2488. Retrieved from https://doi.org/10.1111/jocn.1327

[19]. Laschinger, H. and Nosko, A. (2015). Exposure to workplace bullying and post-traumatic stress disorder 
symptomology: the role of protective psychological resources. Journal of Nursing Management; 23: 252262. Retrieved from https://doi.org/10.1111/jonm.12122

[20]. Linda, S., \& Trudi, M. (2012). Identification of parental stressors in an Australian neonatal intensive care unit. Neonatal, Pediatrics and Child Health Nursing;15 (2).

[21]. Robbins, A. (2015). Mean girls of the ER: The alarming nurse culture of bullying and hazing. Marie Claire.

[22]. Mohebifar, R., and Alijanzade, M. (2015). Studying patient safety culture from the viewpoint of staffs in educational hospitals in Tehran City. Journal Health Safety Workplace bullying; 5(1):57-64.

[23]. Salavati, S., Fanoosi, T., Dehghan, D., and Tabesh, H. (2013). Nurses' perspectives on patient safety culture. Iran Journal Nursing; 26(84):24-33.

[24]. María, C., Davenporta, M., Paula, A., Domíngueza, M., Juan, P., Ferreiraa, M., Ana, L., Kannemanna, M., Agustina, D., Fernando, A., and Torresa, M. (2017), Measuring adverse events in pediatric inpatients with the Global Trigger Tool. Archivos Argentinos de Pediatria; 115(04).

[25]. Pasley, S. (2018). Confronting bullying in the nursing profession. Retrieved from media@bravadohealth.com

[26].Thomas, S. \& Burk, R. (2009). Junior nursing students experiences of vertical violence during clinical rotations. Nursing Outlook; 57, 226-231.1

[27]. Samuels, A., (2015). Workplace Bullying Among Nurses at Psychiatric Hospital in the Western Care. A Mini-thesis submitted in partial fulfilment of the requirements for the Degree of Magister Curationis in The School of Nursing, Faculty of Community and Health Sciences, University of the Western Cape.

[28]. Koontz, V., (2003). Parental Satisfaction in a Pediatric Intensive Care Unit. Theses, Dissertations and Capstones. 695.

[29]. Sochalski, J. (2001). Quality of care, nurse staffing and patient outcomes Policy Polit Nursing Practice; 2(1):9-18.

[30]. American Nurses Association (ANA), (2000). Nurse Staffing and Patient Outcomes in the Inpatient Hospital Setting, Report. Washington, DC: ANA.

[31]. Ortega, A., Christensen K., Hogh A., Rugulies, R. \& Borg, v. (2011). One - year prospective study on the effect of workplace bullying on long - term sickness absence. Journal of Nursing Management; 19, 752759.

[32]. Y1ldırım, Y. (2009). Bullying among nurses and its effects. International Nursing Review; 56, 504-511.

[33]. Heather, K. \& Laschinger, S. (2014). Impact of Workplace Mistreatment on Patient Safety Risk and NurseAssessed Patient Outcomes. The Journal of Nursing Administration, 44, (5), 284-290.

[34]. Meng, (2016). Management of work place bullying in hospital: A review of the use of cognitive rehearsal as an alternative management strategy. Chinese Nursing Association, Retrieved from http://creativecommons.org/licenses/by-nc-nd/4.0/

[35]. Laschinger, H., Wong, C., Regan, S., Young-Ritchie, C. and Bushell, P. (2013).Workplace incivility and new graduate nurses' mental health: the protective role of resiliency. Journal Nursing Administration; 43(78):415-421.

[36]. Lewis, P .and Malecha, A. (2011). The impact of workplace incivility on the work environment, manager skill, and productivity. Journal Nursing Administration; 41(7/8):S17-S23.

[37]. Chipps, E., Stelmaschuk, S., Albert, N., Bernhard, L. and Holloman, C. (2013).Workplace bullying in the OR: results of a descriptive study. AORN Journal; 98(5):479-493.

[38]. Aiken, L., Sermeus,W. and Vanden, H et al., (2012). Patient safety, satisfaction, and quality of hospital care: cross sectional surveys of nurses and patients in 12 countries in Europe and the United States. BMJ Journal; 344.

[39]. Hutchison, M. and Jackson, D. (2013). Transformational leadership in nursing: towards a more critical interpretation. Nursing Inquiry Journal. 20(1):11-22.

[40]. Yildirim, D. (2007) Mobbing in the workplace by peers and managers: mobbing experienced by nurses working in health care facilities in Turkey and its effect on nurses. Journal of Clinical Nursing, 16, 14441453

[41]. Pai, H., and Lee, S. (2011). Risk factors for workplace violence in clinical registered nurses in Taiwan. Journal Clinical Nursing; 20(9e10):1405e12. 